\title{
A STUDY ON THE THERMODYNAMIC PROPERTIES OF ASSOCIATED SOLUTIONS BY LINEAR ASSOCIATION MODELS *
}

\author{
Tomoshige NITTA AND TAKAshi KATAYAMA \\ Department of Chemical Engineering, Osaka University, Toyonaka, Japan
}

\begin{abstract}
The excess thermodynamic properties of associated solutions are discussed by using the linear association models (I) and (II). The linear association model (I) deals with the self-associated system by making distinctions between the association constant and association energy of dimer formation and those of polymer formations. The results of the calculation show that the distinguishing effects are relatively small in quality. The linear association model (II) is proposed as one of the models for the system having the species associated with like and unlike molecules. A characteristic of this model is the existence of a maximum point on the activity coefficient curve. Good results are obtained in the application of model (II) to correlate and interpret the vapor-liquid equilibria for binary mixtures of alcohols and interacting solvents.
\end{abstract}

\section{Introduction}

Vapor-liquid equilibrium is one of the most fundamental properties required for separation column design in chemical processes, and many experiments have been made for getting the equilibrium data and for determining nonidealities of both phases. For estimation and correlation of vapor-liquid equilibrium relations, the factors producing the nonidealities of both phases must be analyzed qualitatively and quantitatively.

For vapor-liquid equilibria at atmospheric and low pressures, deviations from ideal relations come mainly from the liquid phase. Some kinds of theories, such as regular solution theory, have been applied to illustrate the liquid phase nonidealities and it is found that they are successfully applied for slightly nonideal solutions but not for strongly nonideal solutions.

Among many considerable factors producing the large nonideality of liquid solutions, we focussed our attention on strong interactions between molecules, known as association phenomena, because the associated substances such as alcohols are widely utilized in industry and the nonideality of such systems is generally large and sometimes shows peculiar behavior. For instance, the activity coefficient for the system ethanolchloroform $^{31}$ ) has a maximum value and the excess

\footnotetext{
* Received on September 10, 1971

Partly presented at the 34th Annual Meeting of the Soc. of Chem. Engrs., Japan, at Tokyo, April 2, 1969
}

enthalpy plot for the system propanol-pyridine ${ }^{8)}$ shows an $S$-shape, etc.

The solution theories which have been developed to interpret the nonideality of associated solutions can be classified into two groups. One is associated solution theories which treat the associated complexes as new, distinguishable and independent molecular species and are in the wake of Dolezalek ${ }^{13)}$. The other is Barker's theory ${ }^{2)}$ in which the surface of a molecule is divided into several contact points having specific interaction energies with the parts of surrounding molecules and the intermolecular pairs are treated as distinguishable and independent "species" by means of an assumption of quasi-chemical equilibrium. In this theory, the chemical interaction is treated as larger in quality than the physical interaction.

In the associated solution theories many proposals have been made for the definition of association constants (cf. mole fraction, volume fraction, molar density, etc.) and for the assumption of their concentration dependence. Prigogine and coworkers ${ }^{26)}$ first presented theoretical foundations for these treatments in terms of partition function based on quasi-lattice theory, but the physical interaction term was neglected in their paper.

The continuous linear association model has been applied successfully to interpret the thermodynamic properties for alcohol-hydrocarbon systems ${ }^{12,14,16,28 \sim}$ ${ }^{30,35)}$. Recently a hypothesis of a cyclic dimer for alcohol has attracted special interest ${ }^{12,14)}$, although Liddel and Becker ${ }^{18)}$ had already emphasized it following a spectroscopic interpretation. However, the 
effects of cyclic dimer formation on the thermodynamic properties have not been discussed in a general form.

From the viewpoint of molecular structure, selfassociated substances have a tendency to be associated with unlike molecules, but few papers $^{3,10,36)}$ have analyzed the thermodynamic properties for these systems. Kretschmer and Wiebe ${ }^{16)}$ outlined briefly a procedure for extending their model to interacting solvents. Recently Wiehe and coworkers ${ }^{36)}$ extended the Wiehe-Bagley model to interacting solvents and applied it to many alcoholic solutions.

In this paper we first summarize an associated solution theory which includes the physical interaction term by using the concept of a statistical thermodynamic model. The concept has elucidated the assumptions of this theory and enabled the extension of a continuous linear association model to more complicated systems. Our final equations are, however, essentially the same as those of Kretschmer and Wiebe. By use of these equations, the effects of cyclic dimer formation on the thermodynamic properties are calculated and discussed in a general form. Next, this model is extended to mixtures which involve interacting solvents (model II). The behavior of this model is characterized by the possibility of the existence of a maximum point on the activity coefficient curve. Finally, applications of model (II) were made satisfactorily to binary mixtures of alcohols and interacting solvents.

\section{Fundamental Equations}

In an associated solution, the interaction energy by specific orientation is much greater than that by physical interaction. Therefore the associated complexes could be treated as new, distinguishable molecular species; so even pure liquid is treated as a mixture of monomer and polymers if it is self-associated.

Using the cell model for the liquid state, the partition function of the system containing $N_{i}$ molecules of species $i(i=1,2, \ldots)$ is given ${ }^{25)}$ by

$$
Q=\omega\left\{\prod_{i} \phi_{i}^{N_{i}}\right\} \exp \left(-E_{0} / k T\right)
$$

The combinatory factor $\omega$ depends on both the molecular sizes and shapes, and on the relative strengths of the interaction energies. The shape of the linearly associated complexes which are discussed in this paper is a one-dimensional structure as shown in Fig. 1. For a system composed of such complexes, the combinatory factor derived by Flory ${ }^{9)}$ can be applied, ${ }^{26)}$ provided each complex distributes randomly.

$$
\omega=\frac{1}{\prod_{i}\left(\sigma_{i}^{\left.N_{i} N_{i} !\right)}\right.} \cdot\left(\frac{z-1}{\sum_{i} \nu_{i} N_{i}}\right)^{\sum_{i}\left(\nu_{i}-1\right) N_{i}} \cdot\left(\sum_{i} \nu_{i} N_{i}\right) !
$$

From the assumption of random mixing, lattice energy $E_{0}$ may be expressed by a Scatchard-Hildebrand type equation, Eq.(3),

\section{(a) (A)-(A)-(A)-(A)-(A) \\ (b) (A)-(A)-(A)-(A)-(B)}

Fig. 1 Linear association models

$$
E_{0}=-\sum_{i} N_{i} \xi_{i}\left(\sum_{j} \beta_{i j} \Phi_{j}\right)
$$

where $\beta_{i j}$ is the physical cohesive energy density of the $i-j$ pair, and $\xi_{i}$ and $\Phi_{i}$ are the molecular volume and volume fraction of species $i$, respectively.

That is, the fundamental equation of the linear association model is the polymer solution theory derived by Flory. The main assumptions of the theory are summarized as follows,

(1) quasi-lattice model of the liquid structure

(2) random-mixing of the associated complexes

(3) linear structure of the associated complexes.

From the Eqs. (1), (2), and (3), the chemical potential of molecular species $i$ and the excess enthalpy of the solution are derived in general equations as Eqs. (A-3) and (A-5) (see Appendix A).

\section{Linear Association Model (I) -System Associ- ated between Like Molecules-}

\section{1 Activity coefficient}

In this model ( $\mathrm{I}$ ), we consider the system composed of two components $\mathrm{A}$ and $\mathrm{B}$, and that only component $\mathrm{A}$ is linearly self-associated. That is, there exist molecular species $\left(\mathrm{A}_{1}, \mathrm{~A}_{2}, \ldots, \mathrm{A}_{i}, \ldots ; \mathrm{B}\right)$, where subscript $i$ denotes associated $i$-mer. We can imagine a selfassociated $i$-mer of A as shown in Fig. 1 (a). To calculate the thermodynamic properties, we make the further two assumptions.

(1) Additivity of monomer volume for associated polymers, that is,

$$
\xi_{A_{i}}=i \xi_{A_{1}}=i \xi_{A}
$$

(2) The similarity of physical cohesive energy densities, that is,

$$
\beta_{A_{i}, A_{j}}=\beta_{A A}, \quad \beta_{A_{i}, B}=\beta_{A B}, \quad \beta_{B, B}=\beta_{B B}
$$

By using these equations, final expressions for the activity coefficients of $\mathrm{A}$ and $\mathrm{B}$ are given in molar densities as follows.

$$
\begin{gathered}
\ln \gamma_{A}=\ln \left(C_{A_{1}} / x_{A} C_{A_{1}}^{\circ}\right)-v_{A}\left(\sum_{i} C_{A_{i}}+C_{B}-\sum_{i} C_{A_{i}}^{\circ}\right) \\
+\left(b v_{A} / R T\right) \Phi_{B}^{2} \\
\ln \gamma_{B}=\ln \left(C_{B} v_{B} / x_{B}\right)+1-v_{B}\left(\sum_{i} C_{A_{i}}+C_{B}\right) \\
+\left(b v_{B} / R T\right) \Phi_{A}^{2}
\end{gathered}
$$

where

$$
b=\beta_{A A}+\beta_{B B}-2 \beta_{A B}
$$

and $C_{A i}^{\circ}$ denotes the concentration of associated complex $i$-mer in pure liquid of A. Eq.(6) and (7) are the same ones derived first by Kretschmer and Wiebe ${ }^{16)}$ and rederived recently by Renon and Prausnitz ${ }^{29)}$. 


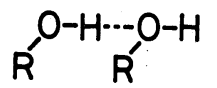

(a)

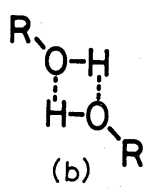

(b)
Fig. 2 Open (a) and cyclic (b) dimers of alcohols

The physical interaction terms which include parameter $b$ in Eqs. (6) and (7) are the same equations as derived by the solubility parameter theory, that is, quadratic equations of volume fraction.

Distribution of the molecular complexes is determined by the association constants, for which two different definitions, Eqs.(9) and (10), have been used in the framework of lory type solution theory (see

Appendix B).

$$
\begin{array}{r}
K_{A_{i}}^{(C)}=K_{A_{i}} \cdot v_{A}=\frac{C_{A_{i}}}{C_{A_{1-i}} C_{A_{1}}} \\
K_{A_{i}}^{(D)}=\frac{\Phi_{A_{i}}}{\Phi_{A_{1-i}} \Phi_{A_{i}}} \cdot \frac{1}{e}
\end{array}
$$

The points of discussion on the linear association model (I) are essentially these two;

(1) Which one is more constant $K_{A_{i}}^{(C)}$ (or $K_{A_{i}}$ ) or $K_{A_{i}}^{(D)}$ for the variation of the number $i$ ?

(2) Is the associated dimer linear or cyclic (Fig. 2)? About the first point, we assume that $K_{A_{i}}^{(C)}\left(\right.$ or $K_{A_{i}}$ ) is independent of the number $i$, as Kretschmer and Wiebe, and Renon and Prausnitz assumed. SaloreaMathot $^{30)}$, and Hwa and Ziegler ${ }^{14)}$ discussed the point that the association constant of dimer formation must be distinguished from that of polymer formation for the interpretation of the excess thermodynamic properties of alcoholic solutions. Liddel and Becker ${ }^{18)}$, and recently Van Ness and coworkers ${ }^{34)}$ suggested from the study of infrared spectroscopic data that an alcohol dimer is cyclic (double-bonded). We discuss in this paper what effects appear on the excess thermodynamic properties by making distinctions between the association constant and association energy of dimer formation and those of polymer formations.

\section{2 Determination of the molecular species distribution}

A molecular balance for component $\mathrm{A}$ is given by

$$
C_{A}=\sum_{i} i C_{A_{i}}
$$

As discussed in subsection. 2.1, $K_{A_{2}}^{(C)}$ is distinguished from $K_{A_{i}}^{(C)}(i \geq 3)$ as follows.

$$
\begin{gathered}
K_{A_{3}}^{(C)}=K_{A_{4}}^{(C)} \ldots \ldots \ldots \ldots=K_{A}^{(C)} \\
K_{A_{2}}^{(C)} / K_{A}^{(C)}=q
\end{gathered}
$$

Eq. (11) is rewritten by means of $K_{A}^{(C)}$ and $q$ as

$$
C_{A}=C_{A_{1}}+\frac{q C_{A_{1}}\left(2-K_{A}^{(C)} C_{A_{1}}\right) K_{A}^{(C)} C_{A_{1}}}{\left(1-K_{A}^{(C)} C_{A_{1}}\right)^{2}}
$$

The monomer concentraction $C_{A_{1}}$ is calculated from Eq. (14) remembering that the condition for the convergence of an infinite series, Eq.(15), must be satisfied.

$$
0 \leq K_{A}^{(C)} C_{A_{1}}<1
$$

The total concentration of associated polymers $\sum_{i} C_{A_{i}}$ is

$$
\sum_{i} C_{A_{i}}=C_{A_{1}}+\frac{q K_{A}^{(C)} C_{A_{1}}^{2}}{\left(1-K_{A}^{(C)} C_{A_{1}}\right)}
$$

In the special case of $q=1, C_{A_{1}}$ and $\sum_{i} C_{A_{i}}$ are given as follows.

$$
\begin{gathered}
C_{A_{1}}=\frac{2 K_{A}^{(C)} C_{A}+1-\sqrt{1+4 K_{A}^{(C)} C_{A}}}{2\left(K_{A}^{(C)}\right)^{2} C_{A}} \\
\sum_{i} C_{A_{i}}=\frac{C_{A_{1}}}{\left(1-K_{A}^{(C)} C_{A_{1}}\right)}
\end{gathered}
$$

\section{3 Heat of mixing}

The association enthalpy of $i$-mer formation $\Delta h_{A i}^{*}$ is given as

$$
\Delta h_{A i}^{*}=-R \frac{\partial \ln K_{A_{i}}}{\partial(1 / T)}
$$

To make a distinction between $\Delta h_{A_{2}}^{*}$ and $\Delta h_{A i}^{*}(i \geq 3)$ corresponding to Eqs.(12) and (13), a parameter $\zeta$ is introduced as

$$
\zeta=\Delta h_{A_{2}}^{*} / \Delta h_{A}^{*}
$$

It is considered that in an idealized condition linear dimer will correspond to the case $\zeta=1$, and cyclic dimer to $\zeta=2$. In a real system $\zeta$ will have a value between 1 and 2. Heat of mixing corresponding to the chemical term is given by

$$
\begin{aligned}
h_{c}^{E}=\left(-\Delta h_{A}^{*}\right) & \left\{\sum_{i} \frac{N_{A_{i}}-N_{A_{i}}^{\circ}}{N_{A}+N_{B}}(2-\zeta)\right. \\
& \left.+\frac{N_{A_{1}}-N_{A_{1}}^{\circ}}{N_{A}+N_{B}}(\zeta-1)\right\}
\end{aligned}
$$

The significance of Eq.(21) could be summarized as follows.

(1) Heat of mixing $\left(h_{c}^{E}\right)$ is simply proportional to minus association enthalpy $\left(-\Delta h_{A}^{*}\right)$, if the molecular species distribution is the same.

(2) Heat of mixing is given by dividing the extreme values at $\zeta=1$ and $\zeta=2$ with $\zeta$.

(3) If $\zeta=1$, heat of mixing is explained as the variation of the average association degree of component $\mathrm{A}$, and if $\zeta=2$, as the variation of the monomer fraction of $\mathrm{A}$.

Eq.(21) is expressed in molar density as

$$
\begin{aligned}
h_{c}^{E}= & \left(-\Delta h_{A}^{*}\right) x_{A}\left\{\left(\frac{\sum_{i} C_{A_{i}}}{C_{A}}-\frac{\sum_{i} C_{A_{i}}^{\circ}}{C_{A}^{\circ}}\right)(2-\zeta)\right. \\
& \left.+\left(\frac{C_{A_{1}}}{C_{A}}-\frac{C_{A_{1}}^{\circ}}{C_{A}^{\circ}}\right)(\zeta-1)\right\}
\end{aligned}
$$

The physical interaction term for heat of mixing is given as

$$
h_{p}^{E}=b^{\prime} \Phi_{A} \Phi_{B}\left(x_{A} v_{A}+x_{B} v_{B}\right)
$$

where

$$
b^{\prime}=b-T \frac{d b}{d T}
$$



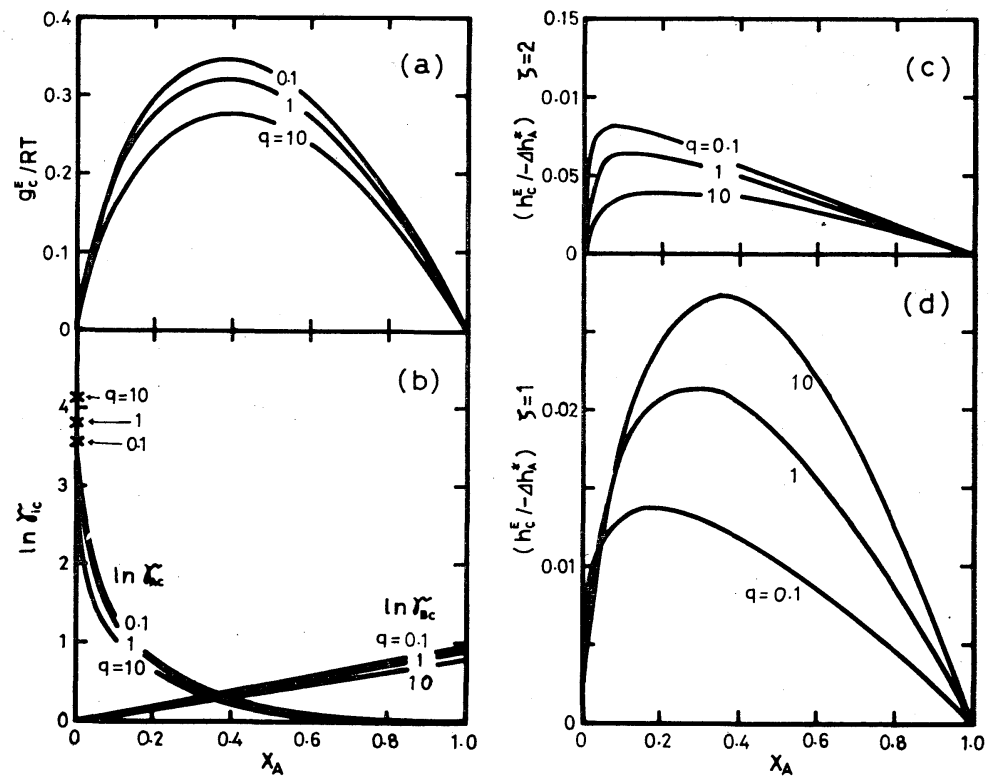

Fig. 3 Calculated properties of liquid solutions by model (I) for $K_{A}=100, v_{B} / v_{A}=1$
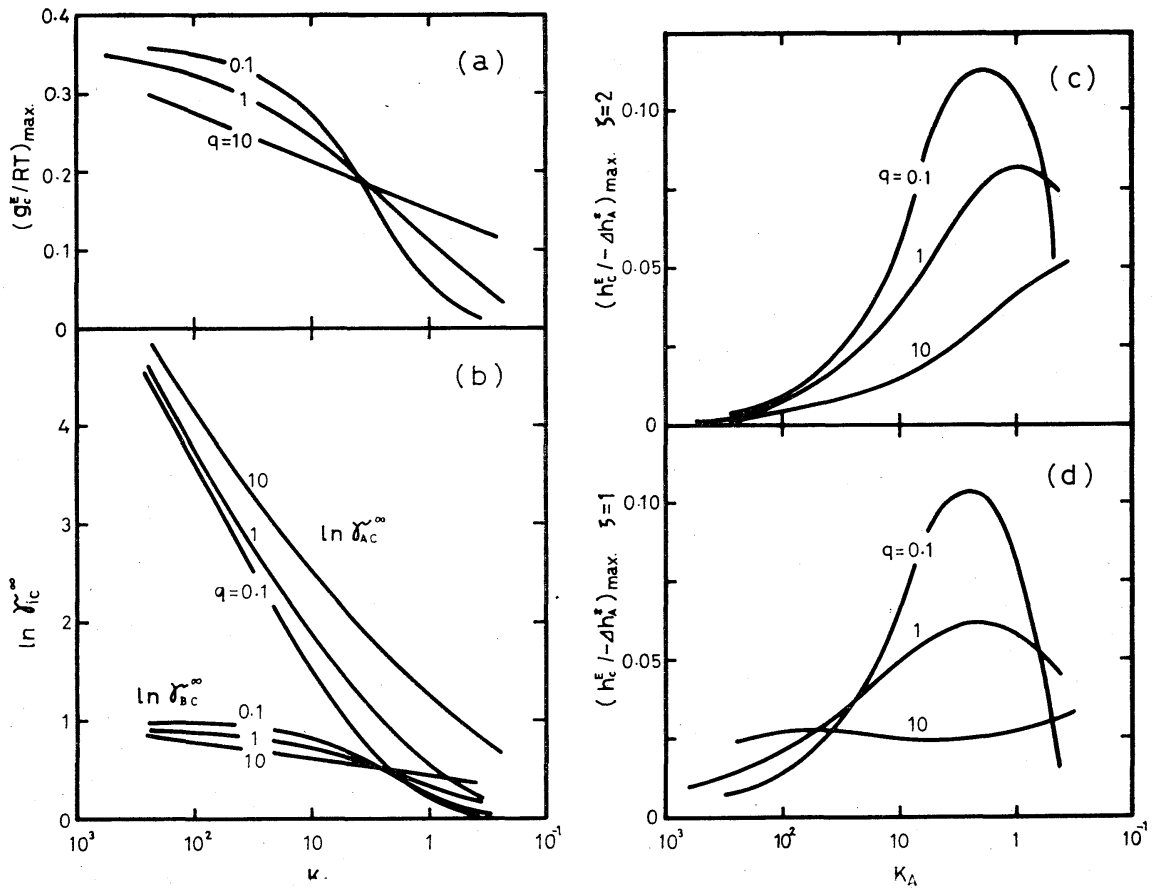

Fig. 4 Calculated maximum properties of liquid solutions by model (I) for $v_{B} / v_{A}=1$

\section{4 Results of the calculation}

Calculations are made taking $K_{A}, q, \zeta$, and $v_{B} / v_{A}$ as parameter. Fig. 3 shows the concentration dependence of the excess thermodynamic properties for $K_{A}=$ $100, v_{B} / v_{A}=1$. In Fig.3(a) the value of $g_{c}^{E} / R T$ decreases with increasing $q$-value. In Fig. 3(b), however, the effects of $q$-value on $\ln \gamma_{A c}$ and $\ln \gamma_{B c}$ are different. Fig.3(c) shows that $h_{c}^{E}$ curves for $\zeta=2$ and $q \geq 0.1$ are highly asymmetric, having a maximum value each at $x_{A} \leq 0.1$, and their magnitude is smaller than that for $\zeta=1$. In Fig. 3(d) the value of $h_{c}^{E} /\left(-\Delta h_{A}^{*}\right)$. for $\zeta=1$ increases with $q$-value. It is noticed that the shape for each case in Figs. 3(a), (b), (c), and (d) is not altered very much by the parameter $q$.

The effects of $q$-value on the magnitude for each property are shown in Fig. 4, in which the maximum values of each property are plotted against association constant $K_{A}$. In Fig. 4(a) lines for each $q$-value cross each other, but the value of $\left(g_{c}^{E} / R T\right)_{\max }$ increases monotonically with increasing $K_{A}$-value (decreasing temperature). A similar result is obtained in Fig. 4(b) for $\ln \gamma_{B c}$, but for $\ln \gamma_{A c}$ lines for each $q$-value do not intersect each other. Fig. 4(c) shows that each curve of $\left(h_{c}^{E} /-\Delta h_{A}^{*}\right)_{\max }$ vs. $K_{A}$ for $\zeta=2$ has a maximum point, which moves to smaller value of $K_{A}$ as $q$-value increases. In Fig. 4(d) for $\zeta=1$, however, the interest- 
ing result is obtained that near $q=10$ the value of $\left(h_{c}^{E} /-\Delta h_{A}^{*}\right)_{\max }$ is almost constant regardless of $K_{A^{-}}$ value. The results calculated by taking the values of $v_{B} / v_{A}$ as $0.5,1$, and 2 show that the magnitude of each property, corresponding to Figs. 3 and 4, increases almost proportionally to the value of $v_{B} / v_{A}$ but the shape changes little.

Although the $\zeta$-value affects considerably the shape and magnitude of the curves for heat of mixing, parameter $q$ has rather little effect on each excess quantity, especially on excess free energy.

\section{Linear Association Model (II) ( $\mathbf{A}_{i} \mathbf{B}_{1}$ Model) - System Associated between Like and Unlike Molecules -}

\section{1 Activity coefficient}

We consider a liquid solution which has the following conditions.

(1) The liquid is made of two components $\mathrm{A}$ and B.

(2) Component A can be linearly self-associated.

(3) Component B is not self-associated.

(4) B molecule can be associated with A monomer and with the end molecule of linearly selfassociated A.

We can imagine the associated species to be shown in Figs. 1(a) and (b), that is, in the solution there exist monomeric and polymeric species $\left(A_{1}, A_{2}, \ldots, A_{i}\right.$, $\left.\ldots ; \mathrm{A}_{1} \mathrm{~B}_{1}, \mathrm{~A}_{2} \mathrm{~B}_{1}, \ldots, \mathrm{A}_{i} \mathrm{~B}_{1}, \ldots ; \mathrm{B}_{1}\right)$.

We make two assumptions as in subsection 2.1.

(1) Additivity of monomer volumes for associated polymers, that is,

$$
\xi_{A i B}=i \xi_{A}+\xi_{B}
$$

(2) The similarity of physical cohesive energy densities, that is,

$$
\begin{aligned}
\beta_{A_{i} B_{1}, A_{j} B_{1}} & =\beta_{A A} \frac{\xi_{A_{i}} \xi_{A_{j}}}{\xi_{A_{i} B_{1}} \xi_{A_{j} B_{1}}}+\beta_{B B} \frac{\xi_{B} \xi_{B}}{\xi_{A i B_{1}} \xi_{A_{j} B_{1}}} \\
& +\beta_{A B} \frac{\xi_{B}\left(\xi_{A_{i}}+\xi_{A_{j}}\right)}{\xi_{A_{i} B_{1}} \xi_{A_{j} B_{1}}}
\end{aligned}
$$

By using these equations, the activity coefficients are expressed by molar densities as follows

$$
\begin{aligned}
\ln \gamma_{A}= & \ln \left(C_{A_{1}} / x_{A} C_{A_{1}}^{\circ}\right)-v_{A}\left(\sum_{i} C_{A_{i}}+\sum_{i} C_{A_{i} B_{1}}+C_{B_{1}}\right. \\
& \left.-\sum_{i} C_{A i}^{\circ}\right)+\left(b v_{A} / R T\right) \Phi_{B}^{2} \\
\ln \gamma_{B}= & \ln \left(C_{B_{1}} v_{B} / x_{B}\right)+1-v_{B}\left(\sum_{i} C_{A_{i}}+\sum_{i} C_{A_{i} B_{1}}+C_{B_{1}}\right) \\
& +\left(b v_{B} / R T\right) \Phi_{A}^{2}
\end{aligned}
$$

where $b$ is given by Eq. (8).

In this model cyclic dimer is assumed absent. We assume further that parameter $q$ for self-association of $\mathrm{A}$ is unity and express the association constant as

$$
K_{A}^{(C)}=C_{A_{i}} / C_{A_{i-1}} C_{A_{1}}
$$

As a site which is available for $B$ molecule is specified at the end of an associated polymer $\mathrm{A}_{i}$, regardless of the number $i$, the association constants for a reaction between unlike molecules are expressed as

$$
K_{A B}^{(C)}=C_{A i B_{1}} / C_{A_{i}} C_{B_{1}}
$$

To clarify the relative strength of like and unlike molecular associations, the dimensionless parameter $r$ is introduced as

$$
r=K_{A B}^{(C)} / K_{A}^{(C)}
$$

We use two dimensionless parameters $K_{A}$ and $r$ to explain the results.

From molecular balances, the simultaneous equations for $C_{A_{1}}$, and $C_{B_{1}}$, are obtained as follows.

$$
\begin{gathered}
C_{A}=\sum_{i} i\left(C_{A_{i}}+C_{A_{i} B_{1}}\right)=\frac{\left(1+K_{A B}^{(C)} C_{B_{1}}\right) C_{A_{1}}}{\left(1-K_{A}^{(\bar{C})} C_{A_{1}}\right)^{2}} \\
C_{B}=\sum_{i} C_{A_{i}}+C_{B_{1}}=\frac{\left(1+K_{A B}^{(C)} C_{A_{1}}-K_{A}^{(C)} C_{A_{1}}\right) C_{B_{1}}}{\left(1-K_{A}^{(C)} C_{A_{1}}\right)}
\end{gathered}
$$

The solution must satisfy Eq.(15). Using $C_{A_{1}}$ and $C_{B_{1}}$ solved, the total concentration of existing complexes is given as

$$
\sum_{i} C_{A_{i}}+\sum_{i} C_{A_{i} B_{1}}=\frac{C_{A_{1}}\left(1+K_{A B}^{(C)} C_{B_{1}}\right)}{1-K_{A}^{(C)} C_{A_{1}}}
$$

\section{2 Heat of mixing}

The equation for heat of mixing by this model is written as

$$
\begin{aligned}
h_{c}^{E}= & \left(-\Delta h_{A}^{*}\right) x_{A}\left\{\frac{\sum_{i} C_{A i}+\sum_{i} C_{A i B_{1}}}{C_{A}}-\frac{\sum_{i} C_{A_{i}}^{\circ}}{C_{A}^{\circ}}\right\} \\
& -\left(-\Delta h_{A B}^{*}\right) \frac{\sum_{i} C_{A i B_{1}}}{\left(C_{A}+C_{B}\right)}
\end{aligned}
$$

where

$$
\Delta h_{A B}^{*}=-R \frac{\partial \ln K_{A B}^{(C)}}{\partial(1 / T)}
$$

Eq. (35) shows that the chemical term of heat of mixing involves two parts, one is the endothermic effect accompanied by breaking the self-association bonding of $\mathrm{A}-\mathrm{A}$, and the other is the exothermic effect by forming the new association bonding A-B. The physical term of heat of mixing is given by Eq.(24).

\section{3 Results of the calculation}

A characteristic of the $A_{i} B_{1}$ model is the existence of a maximum value of $\ln \gamma_{B}$. The composition and the value of $\ln \gamma_{B}$ at a maximum point depend on both $K_{A}$ and $r$. Fig. 5(a) shows the relationship between activity coefficients and the composition for $K_{A}=100$ and $v_{B} / v_{A}=1$. In this case a maximum point appears when $r$ is smaller than 0.4 . In general, when the value of $r$ increases from zero, the activity coefficient decreases and then a maximum point appears in $\ln \gamma_{B}$. By further increasing $r$, the maximum value of $\ln \gamma_{B}$ decreases and its composition moves toward smaller value of $x_{A}$. If $r$ increases still more, the maximum point disappears and both activity coefficients become smaller than unity. It is interesting that when the maximum point appears in $\ln \gamma_{B}$, the value of $r$ does not exceed unity and is smaller for larger $K_{A}$-value. 

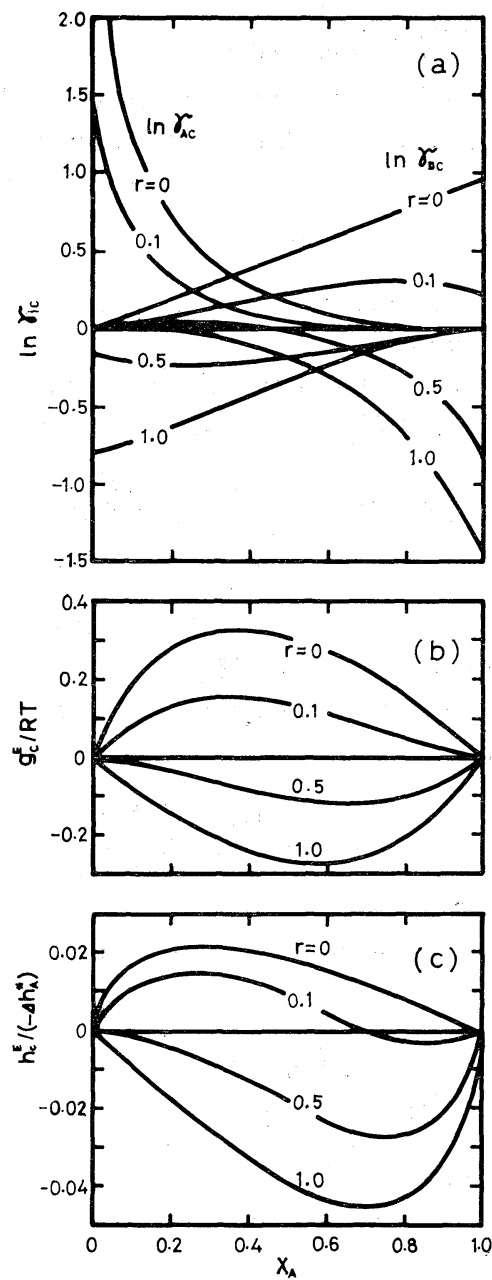

Fig. 5 Calculated properties of liquid solutions by model (II) for $K_{A}=100, v_{B} / v_{A}=1$

Fig. 5(b) shows the relationship between $g_{c}^{E} / R T$ and $x_{A}$ for $K_{A}=100$, and $v_{B} / v_{A}=1$. As the value of $r$ increases, $g_{c}^{E} / R T$ decreases and has a minimum value in $x_{A}<0.5$ at $r=0.5$.

For calculation of the heat of mixing by this model, a value of $\Delta h_{A B}^{*} / \Delta h_{A}^{*}$ must be known in addition to a given set of parameters $K_{A}, r$, and $v_{B} / v_{A}$. In order to know the effects of parameter $r$ on heat of mixing, it is desirable to express $\Delta h_{A B}^{*} / \Delta h_{A}^{*}$ as a function of $r$ $\left(=K_{A B}^{(C)} / K_{A}^{(C)}\right)$.

It is recongnized in general that the larger the association enthalpy $\Delta h^{*}$, the larger the association free energy $\Delta g^{*}(=-R T \ln K)$, but that the relationship between them is not simple. Nevertheless, in order to obtain a general idea of the heat of mixing by this model, we make a very simple assumption,

$$
\Delta h_{A B}^{*}-\Delta h_{A}^{*}=\Delta g_{A B}^{*}-\Delta g_{A}^{*}=-R T \ln r
$$

The absolute value of real $\Delta h_{A B}^{*}$ will usually be smaller than that calculated from Eq.(37), if a correlation ${ }^{23)}$ between enthalpy and entropy of association is considered.
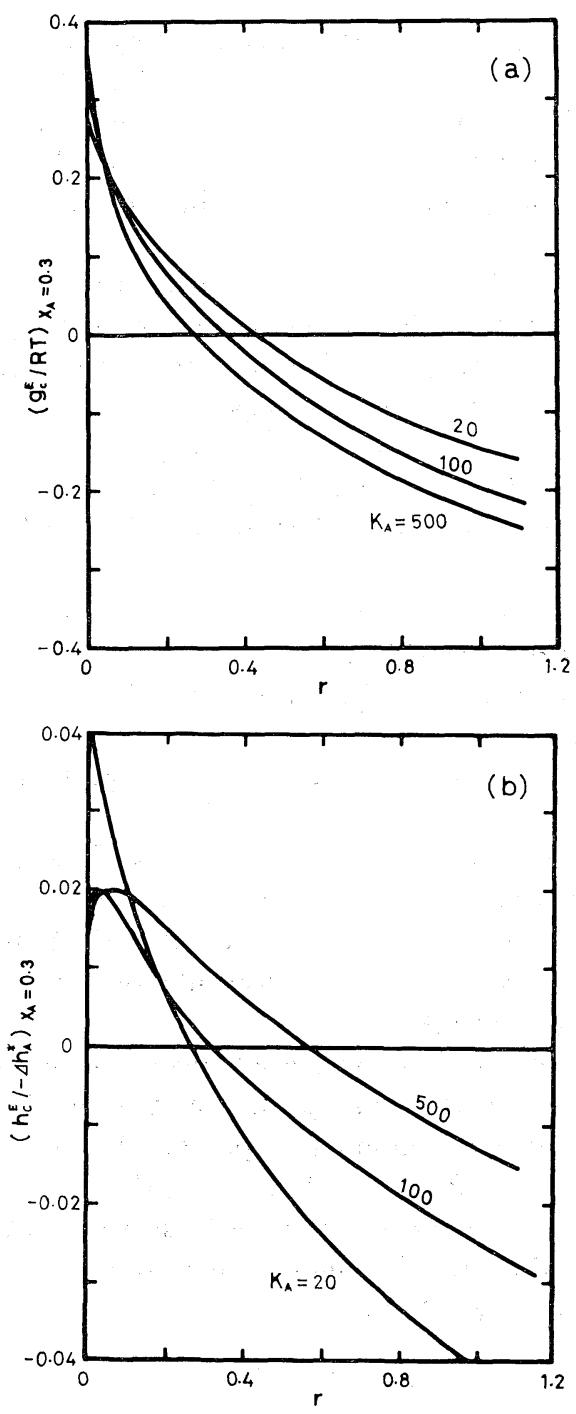

Fig. 6 Calculated properties of liquid solutions at $x_{A}=0.3$ by model (II) for $v_{B} / v_{A}=1$ Dependence of relative strength of association

It is shown in Fig. 5(c) that the concentration dependence of $h_{c}^{E} /\left(-\Delta h_{A}^{*}\right)$ curves is similar to that on $g_{c}^{E} / R T$ in Fig. 5(b). However $r$ dependence of each property is different, as shown in Figs. 6(a) and (b). In Fig. $6(\mathrm{a}), g_{c}^{E} / R T$ at $x_{A}=0.3$ decreases monotonically for increasing $r$, but in Fig. 6(b) each curve of $h_{c}^{E} /\left(-\Delta h_{A}^{*}\right)$ vs. $r$ has a maximum point at a large $K_{A}$-value. This means that when there are chemical interactions between unlike molecules, the number of association complexes between like molecules decreases and the resulting endothermic effect could predominate over the exothermic effect caused by forming association complexes between unlike molecules, and overall the heat of mixing calculated with a set of parameters $K_{A}$ and $r$ could be greater than that with $r=0$. This result will be facilitated by the circumstances that in a real system the absolute value of $\Delta h_{A B}^{*}$ will be smaller than that calculated from Eq. (37) as described. 
Table 1 List of parameters for isothermal vapor-liquid equilibrium data of binary mixtures of alcohols and interacting solvents

\begin{tabular}{|c|c|c|c|c|c|c|c|}
\hline \multicolumn{2}{|r|}{ System } & \multirow{2}{*}{$\begin{array}{c}T \\
{\left[{ }^{\circ} \mathrm{K}\right]}\end{array}$} & \multirow{2}{*}{$\begin{array}{l}K_{A^{\mathrm{a}}} \\
{[-]}\end{array}$} & \multirow{2}{*}{$\begin{array}{c}r \\
{[-]}\end{array}$} & \multirow{2}{*}{$\begin{array}{c}b \\
{[\mathrm{cal} / \mathrm{cc}]}\end{array}$} & \multirow{2}{*}{$\begin{array}{c}\text { Std. Dev.*) or } \\
\text { Av. Dev.**) } \\
{[\%]}\end{array}$} & \multirow{2}{*}{$\begin{array}{c}\text { Reference } \\
\text { of data }\end{array}$} \\
\hline & $(\mathrm{A})$ & & & & & & \\
\hline & MeOH-Benzene & 308.15 & 709 & 0.0413 & 6.00 & $1.56 *$ & $(32)$ \\
\hline & EtOH-Benzene & 333.15 & 144 & 0.0585 & 4.65 & $0.78 *$ & (33) \\
\hline & $n$-PrOH-Benzene & 318.15 & 104 & 0.0687 & 4.86 & $1.06 * *$ & $(5)$ \\
\hline & $i$-PrOH-Benzene & 318.15 & 69.5 & 0.108 & 6.30 & $2.03 * *$ & $(4)$ \\
\hline & MeOH-Ethyl ether & 298.15 & 985 & 0.202 & 5.43 & $1.08 *$ & $(1)$ \\
\hline & EtOH-Ethyl ether & 323.15 & 190 & 0.238 & 4.34 & $1.11 *$ & $(21)$ \\
\hline & $\mathrm{MeOH}$-Triethylamine & 313.10 & 607 & 0.384 & 0.832 & $1.17 *$ & $(7)$ \\
\hline & EtOH-Triethylamine & 308.00 & 301 & 0.502 & 2.62 & $1.48 * *$ & $(6)$ \\
\hline & EtOH-Triethylamine & 338.0 & 126 & 0.431 & 2.23 & $3.16 * *$ & $(6)$ \\
\hline & $\mathrm{MeOH}$-Ethyl acetate & 313.15 & 606 & 0.266 & 6.47 & $5.21 * *$ & $(20)$ \\
\hline & EtOH-Ethyl acetate & 313.15 & 256 & 0.902 & 13.57 & $5.14 * *$ & $(20)$ \\
\hline & $i$-PrOH - Ethyl acetate & 313.15 & 80.9 & 1.682 & 15.49 & $1.42 * *$ & $(20)$ \\
\hline & $\mathrm{MeOH}$-Acetone & 293.15 & 1170 & 1.091 & 13.45 & $1.61 *$ & $(19)$ \\
\hline & EtOH-Acetone & 305.15 & 330 & 3.838 & 30.25 & $0.97 *$ & $(11)$ \\
\hline & $\mathrm{MeOH}$-Dimethyl sulfoxide & 313.15 & 606 & 4.130 & 13.90 & $5.02 * *$ & $(27)$ \\
\hline$* 2$ & Standard Deviation in $\left(\left\langle P_{c a l}-\right.\right.$ & $\left.P_{o b s}\right) / I$ & & & & & \\
\hline$* *)$ & Average Deviation in $\left\{\left(\left(\gamma_{A} / \gamma_{B}\right)\right.\right.$ & cal -( & $\|(\gamma A \mid \gamma$ & & & & \\
\hline a) & Value given by Renon and Pra & & & & & & \\
\hline
\end{tabular}

\section{Discussion}

In the linear association model (I) the effects of formation of cyclic dimers on the excess thermodynamic properties were treated. For alcohol-hydrocarbon solutions Hwa and Ziegler ${ }^{14}$ and Haskel, Hollinger, and Van Ness ${ }^{12)}$ made curve fitting by use of their reliable $g^{E}$ and $h^{E}$ data to determine parameters $K_{A}, \Delta h_{A}^{*}, q, \zeta$, and $b$ (Van Ness, et al. used ideal associated solution theory). However, it is doubtful if it is possible to obtain a set of parameters having significant meanings, since the adjustable parameters to be determined are too many and a good representation of both $g^{E}$ and $h^{E}$ by this simple theory may not be expected. If other information (such as spectroscopic data) about the quantitative relationships between association parameters are available, this work will have more meaning in a practical sense.

In the linear association model (II) it is assumed that cyclic dimer is absent in the solution and that each self-associated polymer has an active site for $\mathrm{B}$ molecules. In an extremely contrary case where all self-associated polymers except monomers have no active site (proton) for $B$ molecule, $A_{1} B_{1}$ type is the only associated structure between unlike molecules. For comparison of the models $\mathrm{A}_{i} \mathrm{~B}_{1}$ and $\mathrm{A}_{1} \mathrm{~B}_{1}$, the activity coefficients are calculated for the "Model $\mathrm{A}_{1} \mathrm{~B}_{1}$ " in which the existence of continuously associated species of $\mathrm{A}$ is assumed, that is, $\mathrm{A}_{1}, \mathrm{~A}_{2}, \ldots, \mathrm{A}_{i}, \ldots$; $A_{1} B_{1} ; B_{1}$. The results of the calculation for the conditions of $K_{A}=100, q=1$, and $v_{B} / v_{A}=1$ are shown in Fig. 7. In this case a maximum point appears on $\ln \gamma_{A}$ curve, and not on $\ln \gamma_{B}$ curve. It is interesting that the thermodynamic properties are significantly affected by what species exist in the associated system. In the binary mixtures of alcohols and interacting solvents

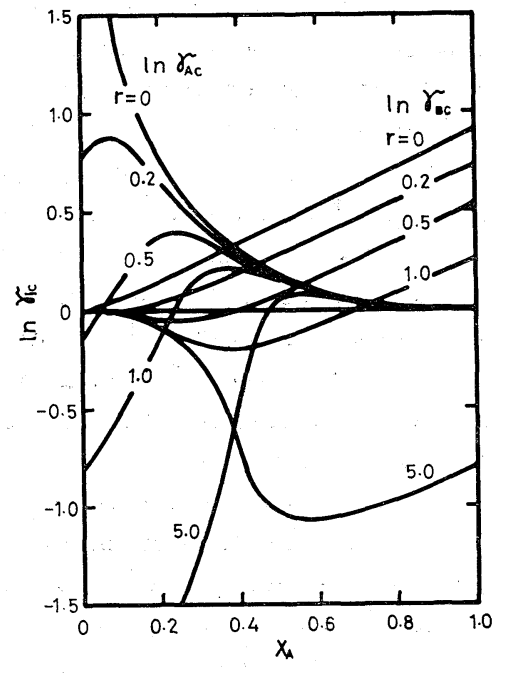

Fig. 7 Calculated properties of liquid solutions by model $A_{1} B_{1}$ for $K_{A}=100, v_{B} / v_{A}=1$

the authors have not found a mixture having such activity coefficients as shown in Fig. 7.

As substances which have a proton acceptor in a molecular constituent and have scarcely the ability of self-association, we could regard aromatics, nitriles, ethers, acetates, ketones, amines, etc. Table 1 shows some results of application of the $\mathrm{A}_{i} \mathrm{~B}_{1}$ model to the vapor-liquid equilibrium data for binary mixtures of alcohols and interacting solvents. The values of selfassociation ' constant $K_{A}$ used for alcohols are those given by Renon and Prausnitz. ${ }^{29)}$ Least-square fitting techniques were used to obtain $r$ and $b$. In these calculations vapor-phase nonidealities were taken into account by using the empirical correlation for second virial coefficients given by O'Connell and Prausnitz.22) Although some objective functions to be minimized were tried, either function $\sum\left(P_{c a l}-P_{o b s}\right)^{2}$ (designat- 


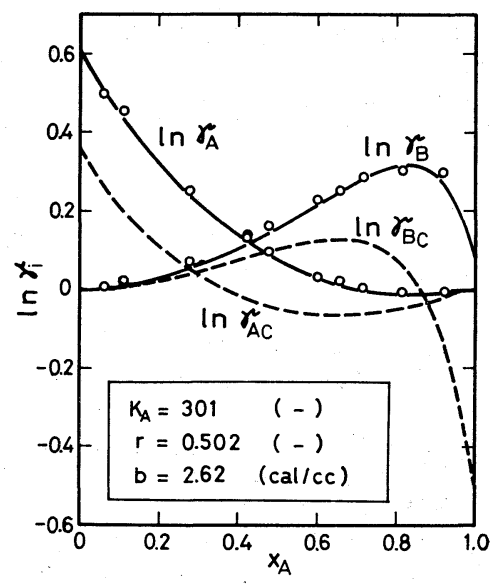

Fig. 8 Activity coefficients for ethanol-triethylamine system at $34.85^{\circ} \mathrm{C}$

ed by $*)$ or $\sum\left(\ln \left(\gamma_{A} / \gamma_{B}\right)_{c a l}-\ln \left(\gamma_{A} / \gamma_{B}\right)_{o b s}\right)^{2}($ by $* *)$ was selected in Table 1. The standard deviation in pressure or average deviation in relative volatility is within the experimental error, as shown in Table 1.

The value of $r$ for the ethanol-benzene system is nearly 0.03 at room temperature, and its temperature dependence is large as expected. Curve fitting for alcohol-benzene systems by the $A_{i} B_{1}$ model reduces slightly the average deviations in comparison with those by model (I). For alcohol-ethyl ether and alcoholtriethylamine systems the correlations of data by $\mathrm{A}_{i} \mathrm{~B}_{1}$ model are satisfactory. Fig. 8 shows the activity coefficients against mole fraction for the ethanol-triethylamine system as an example. The solid lines show the calculated activity coefficients and the dotted lines show those of the chemical term.

For alcohol-acetone systems the values obtained for both $r$ and $b$ are considered to be unreasonably large, which may suggest the necessity of taking into account special interaction between acetone and acetone molecules. The binary mixtures of alcohols and acetates show a similar tendency. For the alcohol-chloroform system the well-known character of having a maximum point on the activity coefficient curve cannot be represented satisfactorily. As a proton of chloroform is estimated to associate with an oxygen atom of alcohol, the association type of $\mathrm{A}_{i} \mathrm{~B}_{j}(j \leq i)$ may be taken into consideration in this case. More theoretical progress would be necessary for the complicated systems of $\mathrm{A}_{i} \mathrm{~B}_{j}$ formation.

Unfortunately the systems containing water cannot be correlated with linear association models, because the structure of self-association of water molecules is not one-dimensional. The extension of associated solution theories in the case of three-dimensional structure of association would be necessary for analyzing the characteristic behavior of aqueous solutions.

\section{Acknowledgement}

Several discussions with Mr. Yoshimasa Sassa on these models are gratefully acknowledged.

\section{Appendix A.}

Derivation of general equations for chemical potential and excess enthalpy by Flory's type solution theory

From Eqs.(1) and (3) in text, chemical potential of molecular species $i, \mu_{i}$, is

$$
\begin{aligned}
\mu_{i} / k T & =-\partial \ln Q / \partial N_{i} \\
& =-\frac{\partial \ln \omega}{\partial N_{i}}-\ln \phi_{i}-\frac{2 \xi_{i}}{k T} \sum_{j} \beta_{i j} \Phi_{j}+\frac{\xi_{i}}{k T} \sum_{l} \sum_{j} \beta_{l j} \Phi_{l} \Phi_{j}
\end{aligned}
$$

From Eq.(2) the derivative of $\ln \omega$ is

$$
\begin{aligned}
& \frac{\partial \ln \omega}{\partial N_{i}}= \ln \frac{N_{i}}{\sum_{j} \nu_{j} N_{j}}+\nu_{i}\left(1-\frac{\sum_{j} N_{j}}{\sum_{j} \nu_{j} N_{j}}\right) \\
&-\left(\nu_{i}-1\right) \ln (z-1)+\ln \sigma_{i}
\end{aligned}
$$

The difference between the chemical potentials of $i$-species at the actual state $\left(\mu_{i}\right)$ and at any reference state $\left(\mu_{i}^{r e f}\right)$ is given as

$$
\begin{aligned}
\frac{\mu_{i}}{k T}-\frac{\mu_{i}^{r e f}}{k T} & =\ln \left(\Phi_{i} / \Phi_{i}^{r e f}\right)-\sum_{j}\left(\frac{\xi_{i}}{\xi_{j}}\right)\left(\Phi_{j}-\Phi_{j}^{r e f}\right) \\
& -2 \frac{\xi_{i}}{k T} \sum_{j} \beta_{i j}\left(\Phi_{j}-\Phi_{j}^{r e f}\right)+\frac{\xi_{i}}{k T} \sum_{l} \sum_{j} \beta_{l j}\left(\Phi_{l} \Phi_{j}-\Phi_{l}^{r e f} \Phi_{j}^{r e f}\right)
\end{aligned}
$$

In the special case that the reference state of $i$-species is pure liquid of $i\left(\Phi_{i}^{r e f}=1\right.$ and $\Phi_{j}^{r e f}=0$, for $\left.j \neq i\right)$, Eq.(A-3) is rewritten as

$$
\begin{aligned}
\frac{\mu_{i}}{k T}-\frac{\mu_{i}^{*}}{k T}= & \ln \Phi_{i}+1-\sum_{j}\left(\frac{\xi_{i}}{\xi_{j}}\right) \Phi_{j}-2 \frac{\xi_{i}}{k T} \sum_{j} \beta_{i j} \Phi_{j} \\
& +\frac{\xi_{i}}{k T} \sum_{l} \sum_{j} \beta_{l j} \Phi_{l} \Phi_{j}
\end{aligned}
$$

The equation of excess enthalpy is given as follows.

$$
\begin{aligned}
H E \cong U E & =-k\left\{\partial \ln Q / \partial(1 / T)-\partial \ln Q^{\circ} / \partial(1 / T)\right\} \\
& =H_{c}^{E}+H_{p}^{E}
\end{aligned}
$$

where

$$
\begin{array}{r}
H_{c}^{E}=\sum_{i}\left(N_{i}-N_{i}^{\circ}\right) \partial\left(-k \ln \phi_{i}\right) / \partial(1 / T) \\
H_{p}^{E}=-\sum_{i} N_{i} \xi_{i} \sum_{j}\left(\beta_{i j}-T \frac{\partial \beta_{i j}}{\partial T}\right) \Phi_{j} \\
+\sum_{i} N_{i} \xi_{i} \sum_{j}\left(\beta_{i j}-T \frac{\partial \beta_{i j}}{\partial T}\right) \Phi_{j}^{\circ}
\end{array}
$$

\section{Appendix B.}

Definition of the association constants and their interrelation

As associated molecules are in thermal equilibrium in a solution, following relation is obtained for $i$-mer formation.

$$
\mu_{A i}-\mu_{A i-1}-\mu_{A_{1}}=0
$$

Combining Eqs.(4), (5), (A-4), and (B-1), the free energy change by association, $\Delta \mu_{A i}^{*}$, is given as

$$
\begin{aligned}
\frac{\Delta \mu_{A_{i}}^{*}}{k T} & \equiv \frac{\mu_{A i}^{*}}{k T}-\frac{\mu_{A i-1}^{*}}{k T}-\frac{\mu_{A_{1}}^{*}}{k T} \equiv-\ln K_{A_{i}}^{(D)} \\
& =-\ln \left(\Phi_{A_{i}} / \Phi_{A_{i-1}} \Phi_{A_{1}}\right)+1
\end{aligned}
$$

where $K_{A_{i}}^{(D)}$ is the "thermodynamically-defined" association constant of $i$-mer formation. Using Eq.(A-1), $K_{A_{i}}^{(D)}$ is given in terms of the cell partition function as

$$
\begin{aligned}
\ln K_{A_{i}}^{(D)}= & \ln \left(\phi_{A_{i}} / \phi_{A i-1}-\phi_{A_{1}}\right) \ln \left(\nu_{A i-1} \nu_{A_{1}} / \nu_{A_{i}}\right)-1 \\
& +\ln (z-1)-\ln \left(\sigma_{A_{i}} / \sigma_{A_{i-1}} \sigma_{A_{1}}\right)
\end{aligned}
$$

The association constant $K_{A_{i}}^{(C)}$ is defined with molar density like Eq.(B-4),

$$
K_{A_{i}}^{(C)}=C_{A_{i}} / C_{A_{i-1}} C_{A_{1}}
$$

We define the dimensionless association constant $K_{A i}$ by means of $K_{A_{i}}^{(C)}$ as

$$
K_{A_{i}}=K_{A_{i}}^{(C)} / v_{A}=\frac{\Phi_{A_{i}}}{\Phi_{A i-1} \Phi_{A_{1}}} \cdot \frac{i-1}{i}
$$

From Eqs.(B-2), (B-3), and (B-4) the interrelation between $K_{A_{i}}^{(C)}$ and $K_{A_{i}}^{(D)}$ is

$$
\begin{aligned}
K_{A_{i}}^{(C)} & =K_{A_{i}}^{(D)} e v_{A}(i-1) / i \\
& =\frac{\psi_{A i}}{\psi_{A i-1} \psi_{A_{1}}} \cdot(z-1) \cdot \frac{\sigma_{A i-1} \sigma_{A_{1}}}{\sigma_{A_{i}}} \cdot \frac{v_{A}}{\nu_{A_{1}}}
\end{aligned}
$$


Wiehe and Bagley ${ }^{35}$ ) used $K_{A_{i}}^{(D)}$, and Kretschmer and Wiebe ${ }^{16)}$, and Renon and Prausnitz ${ }^{29)}$ used $K_{A}^{(C)}$ (or $K_{A}$ ).

The association constant for $A_{i} B_{1}$ formation is obtained in the same manner as,

$$
\begin{aligned}
K_{A_{i} B_{1}}^{(C)} & =K_{A_{i} B_{1}}^{(D)} e v_{B} i /\left(i+\frac{v_{B}}{v_{A}}\right) \\
& =\frac{\psi_{A B_{1}}}{\psi_{A} \phi_{B_{1}}}(z-1) \frac{\sigma_{A} \sigma_{B_{1}}}{\sigma_{A B_{1}}} \cdot \frac{v_{A}}{\nu_{A_{1}}}
\end{aligned}
$$

\section{Nomenclature}

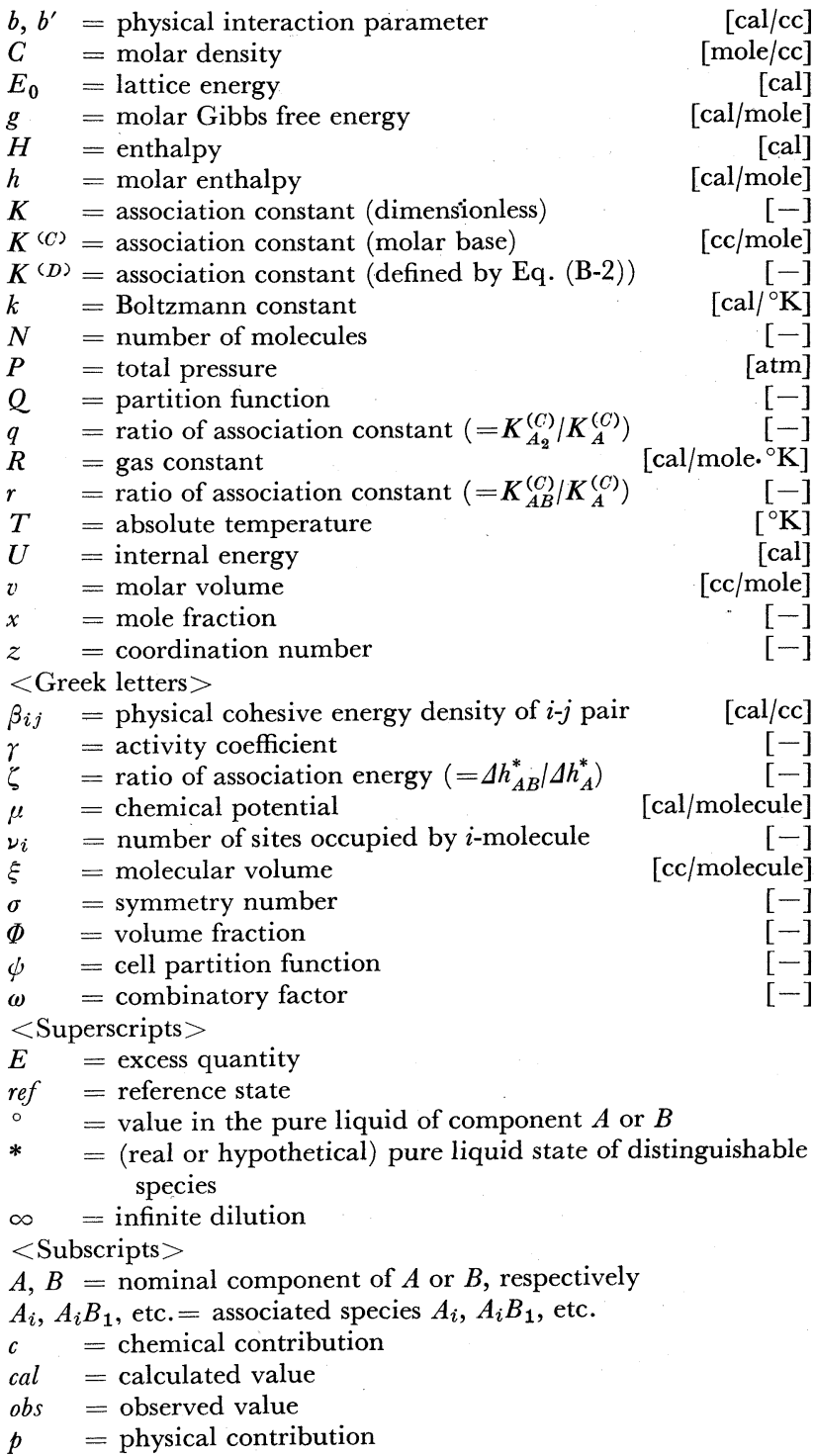

\section{Literature Cited}

1) Arm, H. and D. Bankay: Helv. Chim. Acta, 51, 1243 (1968)
2) Barker, J. A.: J. Chem. Phys., 20, 1526 (1952)

3) Billes, F. and L. Sztraka: Z. Physik. Chem., 235, 286 (1967)

4) Brown, I., W. Fock and F. Smith: Aust. J. Chem., 9, 364 (1956)

5) Brown, I. and F. Smith: Aust. J. Chem., 12, 407 (1959)

6) Copp, J. L. and D. H. Everett: Disc. Faraday Soc., No. 15, 174 (1953)

7) Copp, J. L. and T. J. V. Findlay: Trans. Faraday Soc., 56, 13 (1960)

8) Findlay, T. J. V. and J. L. Copp: Trans. Faraday Soc., 65, 1463 (1969)

9) Flory, P.J.: J.Chem. Phys., 12, 425 (1944)

10) Geiseler, G. and E. Mehnert: Z. Physik. Chem. N. F., 64, 26 (1969)

11) Gordon, A. R. and W. G. Hines: Can. J. Res., B24, 254 (1946)

12) Haskell, R. W., H. B. Hollinger and H. G. Van Ness: J. Phys. Chem., 72, 4534 (1968)

13) Hildebrand, J. H. and R. L. Scott: "The Solubility of Nonelectrolytes", 3rd ed., Reinhold, New York (1950)

14) Hwa, S. C. P. and W. T. Ziegler: J. Phys. Chem., 70, 2572 (1966)

15) Kehiaian, H. and A. Treszczanowicz: Bull. Acad. Polon. Sci. Ser. Sci. Chim., 16, 445 (1968)

16) Kretschmer, C. B. and R. Wiebe: J. Chem. Phys., 22, 1697 (1954)

17) Lacmann, R.: Z. Physik. Chem. N. F., 23, 324 (1960)

18) Liddel, U. and E. D. Becker: Spectrochim. Acta, 10, 70 (1957)

19) Morton, D. S.: J. Phys. Chem., 33, 389 (1929)

20) Murti, P. S. and M. Van Winkle: J. Chem. Eng. Data, 3, 72 (1958)

21) Nagai, Y. and N. Ishii: J. Chem. Soc. Japan (Ind.Chem. Sec.), 38, 16 (1935)

22) O'Connell, J. P. and J. M. Prausnitz: Ind. Eng. Chem., Process Design Develop., 6, 245 (1967)

23) Pimentel, G. C. and A. L. McClellan: "The Hydrogen Bond," W. H. Freeman Co., San Francisco, Calif. (1960)

24) Prausnitz, J. M., C. A. Eckert, R. V. Orye and J. P. O’Connell: "Computer Calculations for Multicomponent VaporLiquid Equilibria", Prentice-Hall, Inc., Englewood Cliffs, New Jersey (1967)

25) Prigogine, I.: "Molecular Theory of Solutions", NorthHolland, Amsterdam (1957)

26) Prigogine, I., V. Mathot and A. Desmyter: Bull. Soc. Chim. Belg., 58, 547 (1949)

27) Quitzsch, K., H. Ulbrecht and G. Geiseler: Z. Physik. Chem., 234, 33 (1967)

28) Redlich, O. and A. T. Kister: J. Chem. Phys., 15, 849 (1947)

29) Renon, H. and J. M. Prausnitz: Chem. Eng. Sci., 22, 299, Errata, 1891 (1967)

30) Sarolea-Mathot, L.: Trans. Faraday Soc., 49, 8 (1953)

31) Scatchard, G. and C. L. Raymond: J. Am. Chem. Soc., 60, 1278 (1938)

32) Scatchard, G., S. E. Wood and J. M. Mochel: J. Am. Chem. Soc., 68, 1957 (1946)

33) Udovenko, V. V. and L. G. Fatkulina: Zhur. Fiz. Khim., 26, $719(1952)$

34) Van Ness, H. C., J. Van Winkle, H. H. Richtol and H. B. Hollinger: J. Phys. Chem., 71, 1483 (1967)

35) Wiehe, I. A. and E. B. Bagley: Ind. Eng. Chem., Fundam., 6, 209 (1967)

36) Wiehe, I. A., S. Dorai, C. G. Rader and A. Chandrasekhar: Chem. Eng. Sci., 26, 901 (1971) 